\title{
Advancing limb neural prostheses
}

\section{Journal Article}

\section{Author(s):}

Raspopovic, Stanisa (iD)

Publication date:

2020-10-16

\section{Permanent link:}

https://doi.org/10.3929/ethz-b-000448480

Rights / license:

In Copyright - Non-Commercial Use Permitted

\section{Originally published in:}

Science 370(6514), https://doi.org/10.1126/science.abb1073

\section{Funding acknowledgement:}

759998 - Restoring natural feelings from missing or damaged peripheral nervous system by model-driven neuroprosthesis (EC) 


\section{Advancing limb neural prostheses \\ Neurotechnology can overcome communication difficulties between prostheses and amputees \\ By Stanisa Raspopovic}

Although sophisticated upper and lower limb prostheses have been developed, amputees cannot control them intuitively, nor perceive sensations from them. These deficiencies result in serious issues, including risk of falls, decreased mobility, heart fatigue during walking and lower functionality while grasping. Moreover, the prostheses are not perceived by the users as part of their own body (low embodiment), which increases cognitive burden during use or device abandonment. An ideal manmachine interface should enable the effortless bidirectional communication between the user and the prosthesis. Neural prostheses that provide bidirectional interfacing with the residual nervous system exploit the persistence of the central and peripheral neural pathways devoted to motor control and sensing (1). A combination of neurotechnologies recently achieved previously unseen capabilities of prosthesis actuation and sensory restoration but several hurdles need to be overcome prior to widespread use of these devices.

Upper-limb amputees actuate opening and closing of commercial hand prostheses by the contraction of residual muscles, with no possibility to control single digits. Aboveknee amputees exploit their residual hip movements to initiate protheses, which then execute predefined patterns of motion. Together with natural actuation, a physiologically plausible sensory feedback from prosthesis to amputee is missing, forcing users to continuously visually inspect their artificial limbs. The key limitation of prostheses actuation is that users must learn the nonintuitive control strategies (e.g., contracting the biceps to close the hand).

In the case of amputations closer to the hand or foot, the control signals can be extracted from the residual muscles, but when amputations are at the thigh or shoulder level, muscles controlling hand or foot are lost. In these cases, a solution is surgical rerouting of nerves towards the other available muscles, called targeted muscular reinnervation (TMR). After this intervention, the nerves grow into the new muscles and gain the capacity to excite the tissue. When users attempt movement, neural signals contract the chest muscles in the case of shoulderlevel amputees (2) or residual thigh muscles in the case of the above-knee amputation (3) (see the figure). Sensors, placed over the skin capture the electrical signal produced by muscle activity, which is then transformed, into the movement of the robotic arm or leg. Yet, control based on skin surface electrodes $(2,3)$ suffer from instability due to electrode movement or detachment.

Further development of the muscle reinnervation concept led to regenerative peripheral nerve interface approach (4). The distal end of a transected peripheral nerve in the arm stump is sutured into a muscle graft, which are implanted with recording wires. These wires are, in turn, connected to prosthesis controls via cables passing through the skin, which have the potential to become broken or induce infections. This creates a natural amplifier of neural signals of volitional control by transducing them into high-gain myoelectric signals. A high signal-noise ratio of this biointerface enabled high-precision control, even for single finger movements in two amputees (4). To overcome the problems of skin electrodes and percutaneous wires, the tiny implantable myoelectric sensors (IMES), capturing the muscular signals directly, were developed. These sensors were injected into the reinnervated muscles of three above-elbow amputees and conferred long-term functional use (5). Whenever the movement intention of an amputee activates the implanted muscles, IMESs capture and amplify these electrical signals and send them wirelessly to the receiving coil embedded into the prosthesis socket to control the motors of the prostheses. This proof-of-concept needs to be demonstrated in a bigger cohort of patients and will hopefully increase controllable movements.

Alongside actuation, restoring sensory information from the artificial limb to the user is essential for functionality. Natural sensations from the missing extremity can be restored to the brain, inducing so-called phantom sensations, through electrical stimulation of the residual nerves proximal to the amputation. Peripheral nerves contain parallel tubular structures, called fascicles, that transmit different sensations (e.g., touch, vibration) from specific areas of the limb. Several neural interfaces with different geometries and placement have been devel- oped and preliminarily tested in upper limb amputees. The stimulation of residual median and ulnar nerves (which innervate fingers and palm), through implanted intraneural transversal multipolar electrodes (TIMEs) (6) restored tactile feedback from prostheses in four upper arm amputees. Relying on the restored feedback, users controlled the amount of force exerted with the prosthesis and distinguished between objects with different compliances and shapes.

Other neurotechnologies that can restore sensory information include Utah slanted multielectrode arrays (USEAs). These consist of a grid of micrometric stimulating wires of different length that are implanted in peripheral nerve fibers (7) and deliver spatially focused (selective) feedback to hand amputees. In a complementary approach, remarkable stability, of several years (in terms of functionality and biocompatibility), was achieved with flat interface nerve electrode (FINE) (8) implants. FINEs enable a gentle nerve flattening, placing the active contacts in proximity to the inner fascicles without penetrating the nerve like TIMEs and USEAs. Thus, FINEs are easier to implant and cause less nerve damage, but at the cost of higher stimulation current to elicit sensations.

Stimulation of leg nerves is a different challenge because they are bigger than nerves in the arm and skin receptors have different density over the foot-sole skin compared to the palm. Through a meticulous surgery, multiple TIMEs were implanted in the sciatic nerve (which innervates foot and lower leg) and elicited selective foot and leg sensations in three above-knee amputees, augmenting their confidence during walking (9). This improved their mobility on stairs, avoidance of falls, and embodiment of the artificial leg, while diminishing the cognitive load during use. Contrary to upper-limb amputation, leg amputation introduces additional health complications such as increased cardiovascular fatigue and decreased mobility. Two highly disabled, above-knee amputees equipped with intraneural feedback from prostheses experienced several health benefits, such as diminished cardiovascular fatigue and pain, increased mobility over uneven terrains and 
This is the author's version of the work. It is posted here by permission of the AAAS for personal use, not for redistribution. The definitive version was published in Science on Vol. 370, 16 Oct 2020. DOI: 10.1126/science.abb1073

brain load decrease (10).

In these studies, different sensations were restored in amputees, but there is limited evidence for perceptions of limb position, speed, and torque, namely proprioceptive sensations, which are essential for walking. To overcome this in below-knee amputees, a surgical approach connecting in series two opposing muscle-tendon ensembles (an agonist and an antagonist) was developed (11). With this strategy, one muscle contracting and shortening (volitional or electrically activated) induces the stretching of the other in opposing direction. This linked motion permits the natural body receptors embedded in the muscle-tendon to send signals, transmitting information about muscle length, speed and force, which is then perceived by the brain as joint proprioception, and used for precise stair walking. However, transfer of this elegant solution to more disabled above-knee amputees could represent a considerable challenge.

Sensations induced by these different approaches are close-to-natural, but they can be perceived like unpleasant electrical tingling. Time-variable electrical stimulation, precisely defined using computational modelling (12), simulating in-silico the responses of tactile neurons innervating the glabrous (hairless) skin, was preliminarily demonstrated to induce more natural sensations $(7,13)$, and could therefore potentially increase acceptance of these technologies. Indeed, computational modelling could optimize implants geometry and electrical parameters to personalize devices in the future.

Recently, muscular control implants and sensory stimulation were combined in four amputees, showing long-term stability and safety, but with limited insights about sensory benefits or cognitive effects (14). The quantified long-term demonstration of the symbiotic and beneficial use of such a bidirectional approach needs to be proven. Indeed, it needs to be explored if artificial motor and sensory signals together could be intuitively handled by the brain, without sensorimotor conflicts or cognitive overload, hopefully resulting in increased functionality.

Neural prostheses for sensory feedback restoration were connected to the stimulator injecting electrical current into the nerves via percutaneous cables, which increases the probability of infections, and has limited robustness. Thus, fully implantable wireless systems need to be developed. These systems should include several important features: long-term stability and safety of the implants, high battery capacity, stable leads (that currently are prone to breakage) and easy replacement of implants or their parts. Although typical implantable neurostimulators (e.g., pacemakers), have pre-programmed stimulation protocols, with no need for a continuous transcutaneous communication, for prostheses a high burden of information has to be wirelessly transmitted through the skin to enable bidirectional communication. This demands high battery capacity while maintaining a limited implant size necessary for surgical placement, representing an important technological challenge to be solved. Presently, most implants require several hours-long surgeries, and therefore minimally invasive procedures should be developed.

Capturing information external to the limb is mandatory to trigger neurofeedback, but prostheses do not have sensors in robotic fingers or under the prosthetic foot. To respond to this need, research efforts are devoted to the development of prosthetic electronic skin, which should be able to accommodate a high density of sensors over flexible polymeric structures (15). In the future these could be imagined as gloves or socks, with robust and high-resolution sensors, placed over the prostheses to transmit different sensory signals such as pressure, movement, and temperature.

To date, these studies are mainly proofof concepts regarding an increase of quality of life or technological viability of neural prostheses, performed with their own metrics, making it difficult to objectively compare their outcomes. They are not systematic clinical studies of safety and efficacy, which are important to achieve the necessary medical certifications. Globally, the regulatory steps are very demanding and costly, and when accounting for individualization of devices and smaller volume need, the economic cost is potentially very high for the end users. This could hinder the widespread use of these technologies. Public healthcare systems vary considerably from country to country and each carries out its own assessment of health technology to support its decisions regarding reimbursement, mainly by demonstrated costeffectiveness and benefits that increase "Quality-Adjusted Life-Years". Therefore, it is of paramount importance, to plan from the first steps of the bidirectional neural prosthesis tests, how to demonstrate safety and health gains, because this would augment the likelihood of device approval.

The future of neurotechnological intervention for amputees will be in the person- alized and combined use of these technologies. Indeed, depending on the amputation level and patients' characteristics, the customized combination of muscular and sensory interventions is likely to benefit amputees in the long term.

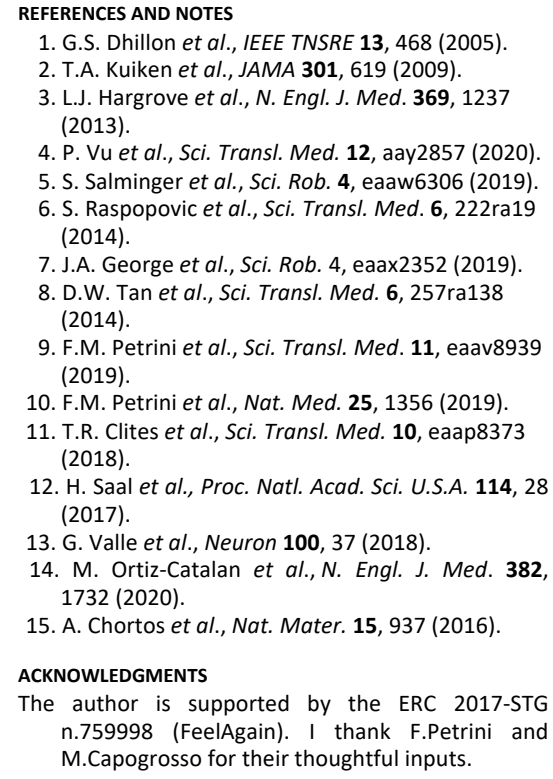

12. H. Saal et al., Proc. Natl. Acad. Sci. U.S.A. 114, 28 (2017).

13. G. Valle et al., Neuron 100, 37 (2018).

14. M. Ortiz-Catalan et al., N. Engl. J. Med. 382, 1732 (2020).

15. A. Chortos et al., Nat. Mater. 15, 937 (2016).

\section{ACKNOWLEDGMENTS}

The author is supported by the ERC 2017-STG n.759998 (FeelAgain). I thank F.Petrini and M.Capogrosso for their thoughtful inputs.

Neuroengineering Lab, DHEST, IRIS, ETH Zurich, Switzerland. Email: Stanisa.raspopovic@hest.ethz.ch 


\section{Bidirectional limb neural prostheses}

Residual motor and sensory neurons in arms and legs of amputees can be used with implants and surgery techniques to confer different sensations and precise motor control of prostheses. Such bidirectional communication and possibly combinations of approaches should improve the quality of life for amputees.

Residual nerve Muscle

Sensors on skin $\square$ Prosthetic device

\section{Targeted muscle reinnervation}

Nerves grow into new muscles, where they can excite the tissue. This is detected by sensors placed on the skin that then move the robotic prosthetic.

\section{Regenerative interface}

Transected peripheral nerves are sutured into a muscle graft and are implanted with myoelectric sensors that are connected to prosthesis motor controls.

\section{Sensory interfacing}

Sensory information, such as tactile feedback, can be restored by using various implants that stimulate electrically residual nerves.

\section{Agonist-antagonist myoneural interface}

Proprioceptive sensations, such as limb position and speed, are important for walking and can be restored in amputees through surgical connection of residual muscles.

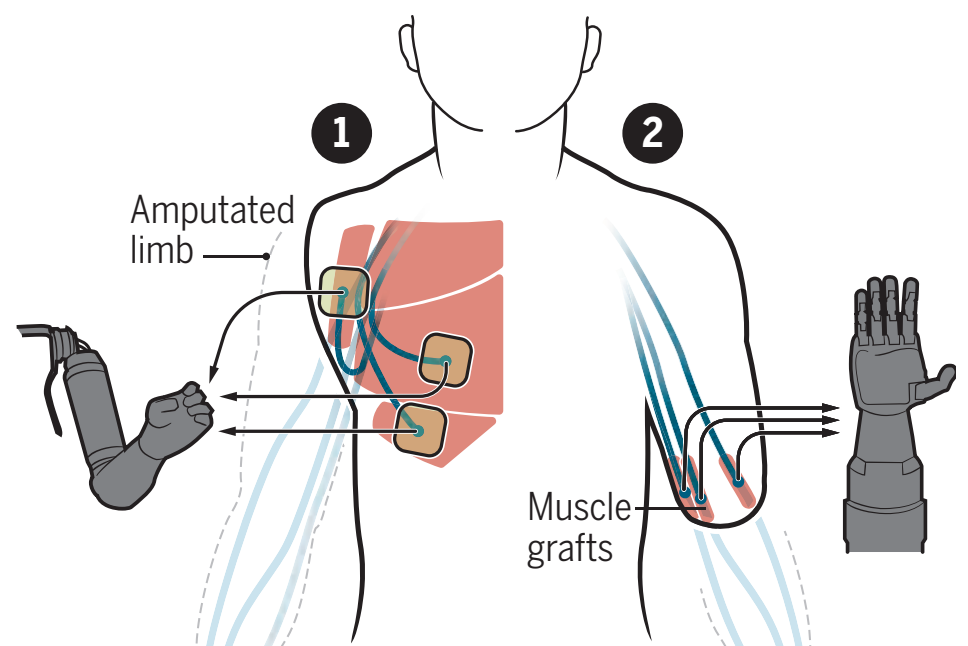

